\begin{tabular}{l|l} 
Revista Educación \\
ISSN: 0379-7082 \\
ISSN: 2215-2644 \\
revedu@gmail.com \\
Universidad de Costa Rica \\
Costa Rica
\end{tabular}

\title{
Empleo de una aplicación informática como estrategia didáctica para el desarrollo de aptitudes académicas
}

Congacha Aushay, Edgar Patricio; Santillán Castillo, Julio Roberto; Guerra Salazar, José Enrique; Barba Vera, Ruth Genoveva

Empleo de una aplicación informática como estrategia didáctica para el desarrollo de aptitudes académicas

Revista Educación, vol. 42, núm. 2, 2018

Universidad de Costa Rica, Costa Rica

Disponible en: http://www.redalyc.org/articulo.oa?id=44055139027

DOI: https://doi.org/10.15517/revedu.v42i2.27472

Esta obra está bajo una Licencia Creative Commons Atribución-NoComercial-SinDerivar 3.0 Internacional. 
Empleo de una aplicación informática como estrategia didáctica para el desarrollo de aptitudes académicas

Use of an Informatic Application as a Didactic Strategy for the Development of Academic Aptitudes

Edgar Patricio Congacha Aushay [1]

Escuela Superior Politécnica de Chimborazo, Ecuador

DOI: https://doi.org/10.15517/revedu.v42i2.27472

renovaspc@hotmail.com

iD http://orcid.org/0000-0002-2043-3378

Julio Roberto Santillán Castillo [2]

Escuela Superior Politécnica de Chimborazo, Ecuador

julio.santillan@espoch.edu.ec

(D) http://orcid.org/0000-0002-4087-6627

José Enrique Guerra Salazar [3]

Escuela Superior Politécnica de Chimborazo, Ecuador

j_guerra@espoch.edu.ec

(iD http://orcid.org/0000-0003-2535-7682

Ruth Genoveva Barba Vera [4]

Escuela Superior Politécnica de Chimborazo, Ecuador

rbarba@espoch.edu.ec

(iD http://orcid.org/0000-0003-0272-171X

Recepción: 18 Diciembre 2016

Aprobación: 04 Enero 2018

\section{ReSUMEN:}

La presente investigación tiene por objetivo observar el impacto que produce el uso de un software educativo con estrategias didácticas dentro del campo de la aptitud académica concerniente al razonamiento numérico, verbal y abstracto en la educación, considerando la teoría cognitiva del diseño instruccional de Robert Gagné, que propicia el desarrollo de habilidades y destrezas en el aprendizaje. Se trabajó con 175 estudiantes que cursan el último año de educación media de cuatro unidades educativas de la ciudad de Riobamba. Las evaluaciones se llevaron a cabo al iniciar y al finalizar la investigación con pruebas similares a las aplicadas para el ingreso a las universidades. Como resultado de la investigación se observó que 155 estudiantes incrementaron su puntuación luego de la preparación con el software, lo cual representó un crecimiento del 94,12\% en el puntaje obtenido, lo que permite concluir que las tecnologías informáticas son herramientas de apoyo para el desarrollo de aptitudes académicas.

\section{NotAS DE AUTOR}

[1] Tecnólogo en Informática Aplicada, Licenciado y Doctor en Ciencias de la Educación Mención Informática Educativa, posee una Maestría en Informática Aplicada a la Educación. Todos estos títulos los obtuvo en la Escuela Superior Politécnica de Chimborazo. Se ha desempeñado como docente de las unidades educativas "Isabel de Godín" y "Santa Mariana de Jesús", de la ciudad de Riobamba (Ecuador), y en la Universidad Estatal de Bolívar (Guaranda, Ecuador). También ha cumplido con las funciones de director del Área de Informática de la Unidad Educativa "Isabel de Godín”, así como coordinador de Proyectos Escolares en la Unidad Educativa "Santa Mariana de Jesús".

[2] Magister en Docencia Universitaria e Investigación Educativa, Doctor en Informática aplicada. Docente titular principal y vicedecano de la Facultad de Informática y Electrónica de la Escuela Superior Politécnica de Chimborazo.

[3] Maestría en Diseño de Sistemas Electrónicos; Magíster en Docencia Universitaria e Investigación Educativa; Ingeniero en Electrónica y Computación. Docente principal de la Facultad de Informática y Electrónica de la Escuela Superior Politécnica de Chimborazo Riobamba, Ecuador

[4] Estudiante de Doctorado en Ingeniería línea de investigación Informática, de la Pontificia Universidad Católica del Perú. Magister en Interconectividad de Redes, Ingeniera en Electrónica y Computación. Técnico Docente de la Facultad de Informática y Electrónica de Escuela Superior Politécnica de Chimborazo Riobamba, Ecuador. 
Palabras Clave: Aptitud académica, aprendizaje, software educativo.

\section{Abstract:}

The objective of this research was to observe the impact that the use of an educational software with educational strategies within the field of academic aptitude in regards to the numerical, verbal and abstract reasoning in education. Robert Gagné s Instructional Design Theory, which fosters the development of learning skills and abilities was used. A total of 175 students enrolled in the senior year at four schools in Riobamba city were evaluated at the beginning and the end of the research with similar tests as the admission tests applied for college entrance. As a result of the investigation, it was observed that 155 students increased their scores after preparing themselves with the software. This represented an increase of $94.12 \%$ in the scores obtained allowing to conclude that computer technologies are tools to support the development of academic aptitudes.

KEYWORDS: Academic aptitude, learning, educational software.

\section{INTRODUCCIÓN}

En el Ecuador, desde el año 2011, para ingresar a una universidad pública, el estudiantado deben rendir un examen que evalúa las aptitudes académicas, concernientes al razonamiento numérico, verbal y abstracto. Con base en el puntaje alcanzado puede acceder a determinadas carreras. En ese año, según el Instituto Nacional de Estadísticas y Censos del Ecuador (INEC), la tasa de matriculación en la educación superior fue de $30,1 \%$, decreciendo en el 2012 a $28,6 \%$ y a 26,6 \% en el 2013 (El universo.com, 2014). El promedio alcanzado sobre 1000 puntos a nivel nacional en las pruebas realizadas en 2012 se ubica en 667, con un $11 \%$ de estudiantes con notas inferiores a 551 puntos. Para 2014, el promedio se incrementa a 710, según la Secretaría Nacional de Educación, Ciencia, Tecnología e Innovación (Senescyt). (El Universo, 2014). Este rendimiento puede considerarse bajo comparado con el puntaje requerido para ingresar a carreras como Ingeniería Civil, cuyos puntajes de asignación oscilan entre 917 y 983 puntos, Ingeniería Comercial que requiere entre 811 y 961, Ingeniería de Sistemas entre 811 a 911, o Medicina con puntajes de 900 a 939 (Secretaría de Educación Superior, ciencia, tecnología e innovación, 2014). Se genera, según encuestas realizadas, una inadecuada selección de la carrera y una frustración en la vida profesional. Este problema puede originarse en la naturaleza de las pruebas, o en la escasa o inadecuada preparación de la población aspirante.

Según INEC, hasta el año 2013, el 43,6\% de la población ya utilizó un computador (ecuadorencifras.com, 2013) y solo el 20,0\% de las personas eran consideradas analfabetas digitales (ecuadorencifras.gob.ec, 2014). Con base en esto, se considera que la mayoría de la población tiene conocimiento en el manejo de equipos informáticos y sus aplicaciones, lo que facilita el empleo de la aplicación informática propuesta en esta investigación, por cuanto no se requieren mayores detalles sobre su uso.

Con estos antecedentes, esta investigación busca demostrar que el empleo de una herramienta informática potencia la inteligencia, memoria y destrezas cognitivas de la persona, para lo cual se empleó un enfoque basado en la teoría del aprendizaje de Robert Gagné (Gargallo, Pérez, Serra, Sánchez y Ros, 2007).

\section{Perspectiva teórica}

Para iniciar el trabajo, fue necesario que el grupo de investigación estableciera algunas definiciones basadas en revisiones bibliográficas y en conceptualizaciones propias, las que se detallan a continuación:

El aprendizaje. Según Robert Gagné, es una secuencia de procesos que inicia al captar la atención del estudiantado, informar el objetivo del aprendizaje, y estimular el recuerdo de los aprendizajes previos. Todo esto se complementa con la presentación del estímulo, guiar el aprendizaje, provocar la conducta, proporcionar retroalimentación, evaluar la ejecución, mejorar la retención y la transferencia (Arancibia, Herrera y Strasser, 2008). 


\section{Modelo instruccional de Robert Gagné}

La teoría del aprendizaje de Gagné está integrada por elementos conductuales y cognitivos, toma de Skinner la importancia a los refuerzos y el análisis de tareas; y de Ausubel, el aprendizaje significativo y la creencia de una motivación intrínseca. Integra con la teoría del desarrollo cognitivo de Piaget y el aprendizaje social de Bandura. Todo explicado por Gagné http://image.slidesharecdn.com/modelosdeinstruccin-10020712 1754-phpapp01/95/modelos-de-instruccin-10-728.jpg?cb=1336075967 en forma sistémica y organizado desde el modelo del procesamiento de la información en dos elementos: las condiciones internas que van a intervenir en el proceso y las condiciones externas que favorecerán un aprendizaje óptimo (Chipia, 2008).

Los diferentes procesos internos que tienen lugar durante el aprendizaje se ven necesariamente afectados por condiciones externas al individuo. Son definidas por Gagné, como aquellos eventos de la instrucción, externos al individuo, que permiten que se produzca un proceso de aprendizaje (Fontalvo, 2008).

\section{Las estrategias didácticas}

tesis, es la elección de las técnicas y actividades necesarias para cumplir los objetivos propuestos, adapt

Son tareas vinculadas a aprendizajes del estudiantado acordes al entorno y a los contenidos sobre los que versan las asignaturas enfocadas en contribuir con la construcción del conocimiento (Peñalosa y Castañeda, 2007).

La estrategia didáctica responde a un procedimiento organizado, formalizado y orientado para la obtención de una meta definida (Boza y Toscano, 2012). Requiere del perfeccionamiento de los procedimientos y las técnicas elegidas y detalladas por el personal docente o el personal diseñador de software educativo. En síntesis, es la elección de las técnicas y actividades necesarias para cumplir los objetivos propuestos, adaptadas al modelo instruccional de Gagné.

\section{La aptitud académica}

Es el conjunto de habilidades intelectuales que permiten la adquisición, elaboración y aplicación de ideas para la resolución de problemas. El objetivo de las pruebas de aptitud académica (PAA) es identificar las habilidades, destrezas y capacidades que posee el estudiantado y que influyen en el desempeño académico y profesional (Valencia, 2017). Son cuestionarios con preguntas cerradas de opción múltiple y exploran aquellos procesos de aprendizaje requeridos.

Las aptitudes académicas se clasifican en:

Razonamiento verbal. Mide las habilidades para comprender conceptos expresados en palabras, evalúa la capacidad para abstraer, generalizar y pensar en forma organizada, es indispensable en carreras como derecho, ciencias sociales, diplomacia, turismo, periodismo, entre otras (Morales, 2015).

Razonamiento numérico. Mide la habilidad para razonar con números, manipular relaciones numéricas y operar inteligentemente con materiales cuantitativos. Evalúa la comprensión de las relaciones numéricas y la facilidad para manejar conceptos numéricos. Es indispensable en carreras como matemáticas, física, química, computación, ingeniería, economía, contabilidad, entre otras (Morales, 2015).

Razonamiento abstracto. Mide la habilidad para percibir entre patrones de figuras abstractas, para generalizar y deducir principios con base en dibujos. Es imprescindible en carreras como arquitectura, medicina, música, filosofía (Morales, 2015). 


\section{Software educativo}

Es todo programa informático elaborado con un fin didáctico, desde aquellos basados en los modelos conductistas de la enseñanza, hasta los programas experimentales de enseñanza inteligente asistida por ordenador (EIAO), que buscan imitar la labor tutorial y personalizada que realiza el profesorado, y alcanzar los objetivos educativos, mejorar la calidad de la enseñanza, reducir costos, incrementar y facilitar el acceso a la educación, entre otros (Cataldi, 2000).

\section{Materiales y métodos}

La población de estudio corresponde a un total de 1104 estudiantes que cursan el tercer año de bachillerato en cuatro unidades educativas de la ciudad de Riobamba. Se trabajó con una muestra calculada de 175 estudiantes en el año lectivo 2014-2015, cuyas actividades se desarrollan en jornada matutina en forma presencial. Se tomó una muestra probabilística, que consiste en un subconjunto donde todos los elementos de la población tienen la misma posibilidad de ser elegidos y su procedimiento de selección es de manera aleatoria o al azar, y los resultados son generalizables a la población (Mantilla, 2015).

$\mathrm{Al}$ inicio de la investigación se aplicaron encuestas para determinar los recursos a través de los cuales el estudiantado apoya su comprensión y capacitación. Para la validación de confiabilidad del instrumento aplicado, se empleó el coeficiente Alfa de Cronbach, en el que se obtuvo un valor de 0.8 , que supera al mínimo aceptable (Bojórquez, López, Hernández y Jiménez, 2014). Se empleó un análisis de pre-estudio y postestudio con estructura similar a las pruebas aplicadas para el ingreso a las universidades, con el fin identificar la situación inicial y final del grupo en estudio y comparar sus resultados.

Como herramienta para la realización de este estudio, se desarrolló una aplicación informática cuyo propósito es contribuir en la preparación de estudiantes en el campo de las aptitudes académicas y evaluar sus resultados (Llosent, 2015), basada en guías de pruebas de aptitudes académicas elaboradas por comités internacionales (Blanco, García, Gómez, Moreira y Romero, 2016).

La evaluación consideró dos parámetros:

- La usabilidad de la aplicación informática, por medio de la aplicación de una encuesta, cuyas preguntas están basadas en la escala de medición específica (EE). Tiene como propósito, al igual que la escala Likert (Fabila y Izquierdo, 2013), medir actitudes y conocer el grado de conformidad del sujeto encuestado, con preguntas cuyas repuestas son categorizadas, según el concepto de interés. El producto de fiabilidad y validez es mucho más alto que las preguntas formuladas con escala Likert $(\mathrm{DA} / \mathrm{ED})^{[5]}$ (Llaurado, 2011).

- El desarrollo de las aptitudes académicas, mediante la aplicación de una prueba similar a la empleada para el ingreso a las universidades, en una etapa de pre estudio en la que se evaluaron las aptitudes académicas que posee el estudiantado antes del empleo de la aplicación, y en la etapa de post estudio, luego de haber empleado la aplicación informática por el lapso de dos meses.

\section{Resultados}

Como un primer paso de la evaluación se analizó la usabilidad del software desarrollado, mediante la aplicación de una encuesta a la muestra del estudio. A continuación, se detallan los resultados obtenidos. 


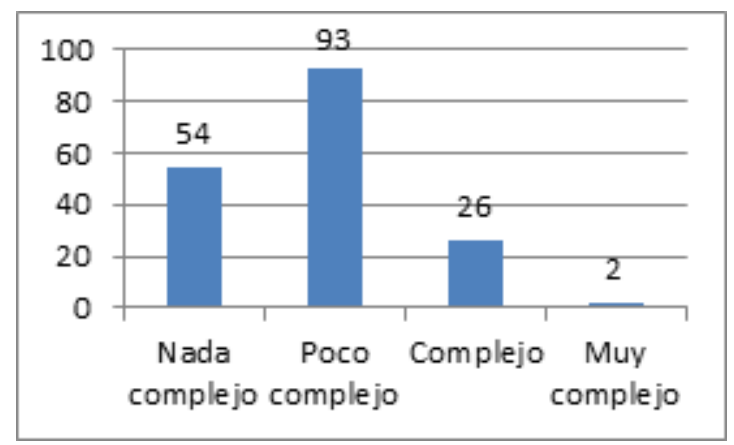

FIGURA 1

Complejidad en el empleo del software desarrollado. Esquema elaborado para la investigación.

En la Figura 1 se analiza la complejidad en el empleo del software desarrollado, en la que se aprecia que 147 estudiantes, que representan el $84 \%$, afirman que el software no presenta complejidad en su empleo, y 28 afirman que existe algún grado de complejidad.

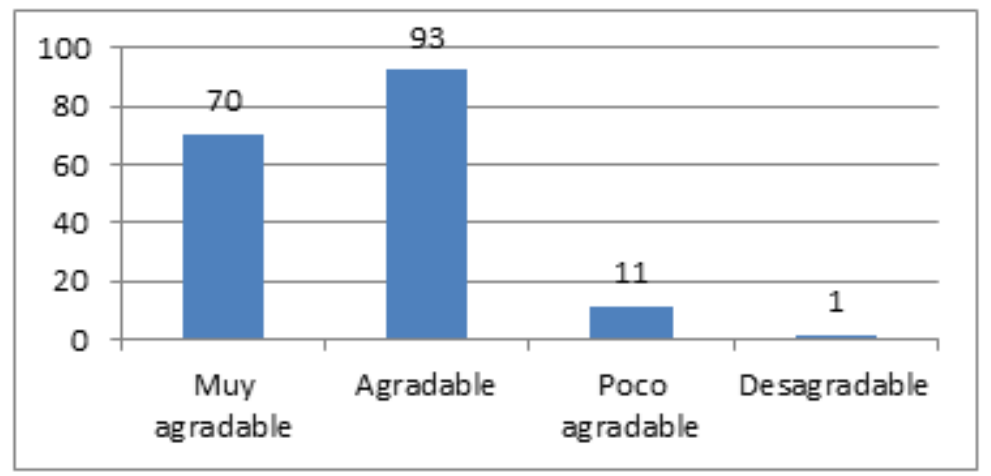

FIGURA 2

Evaluación de la interfaz de la aplicación. Esquema elaborado para la investigación.

En la Figura 2 se presenta el resultado de la evaluación de la interfaz del software desarrollado. El $93 \%$ considera que la interfaz de la aplicación es agradable, y 12 estudiantes muestran algún nivel de desagrado con esta.

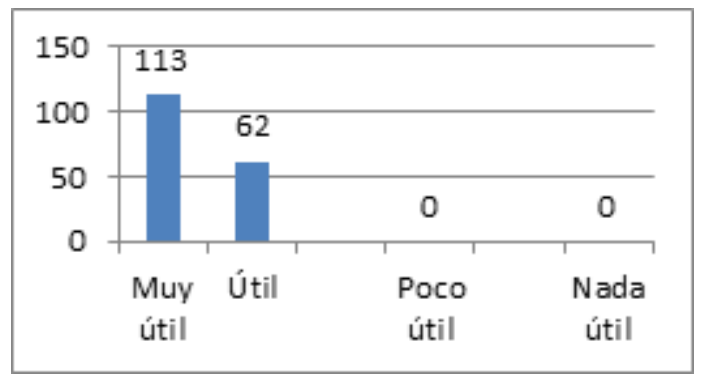

FIGURA 3

Utilidad de la información suministrada por el software desarrollado. Esquema elaborado para la investigación.

En la Figura 3 se aprecian los resultados de la evaluación de los contenidos que despliega la herramienta. Es notorio que el $100 \%$ del estudiantado encuestado encuentra algún grado de utilidad de los contenidos presentados por la herramienta. 
Luego de la aplicación del software desarrollado, se evaluó el impacto que tuvo la información presentada en las aptitudes académicas del estudiantado, según su propia apreciación.

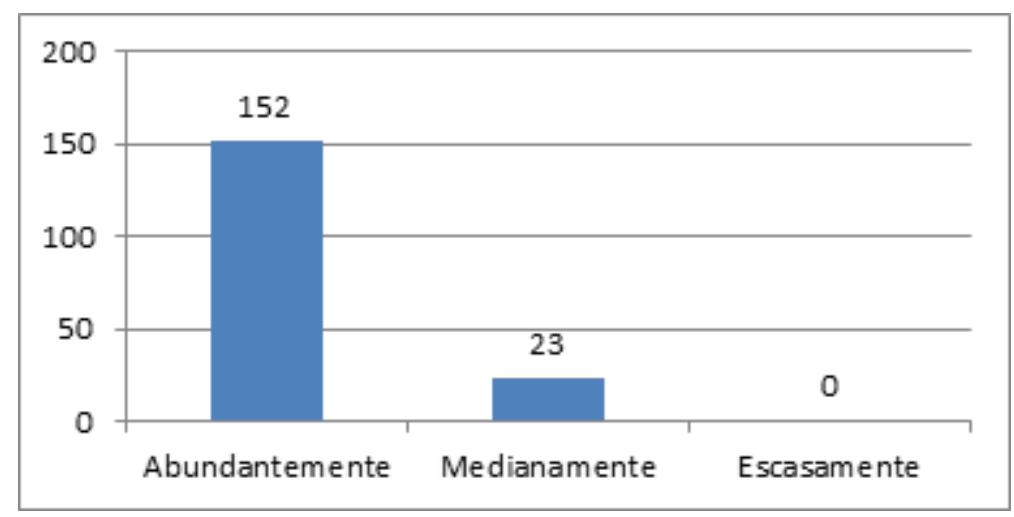

FIGURA 4

Evaluación de la influencia de los contenidos presentados por el software en la atención del estudiantado. Esquema elaborado para la investigación.

En la Figura 4 se observa que el $87 \%$ considera que los contenidos presentados mejoran la atención abundantemente, el $13 \%$ considera que aporta medianamente, y nadie los considera como un aporte escaso.

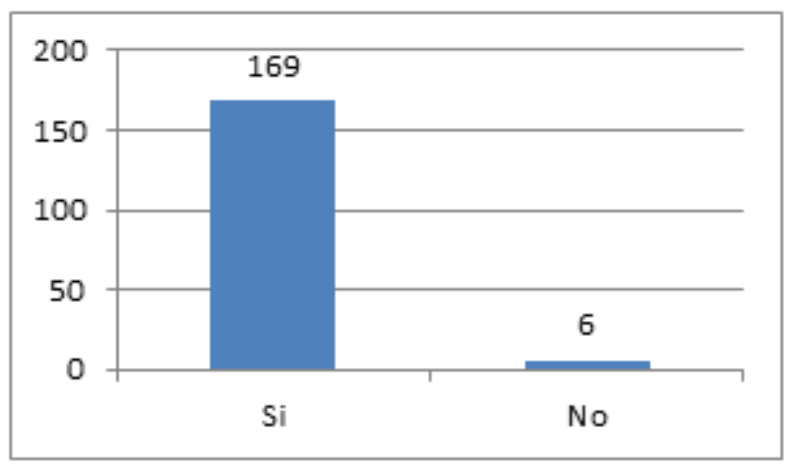

FIGURA 5

Evaluación del aporte del software desarrollado en la preparación académica del estudiantado. Esquema elaborado para la investigación

El aporte de la herramienta desarrollada en la preparación académica del alumnado se representa en la Figura 5, en la que se aprecia que el $97 \%$ piensa que es un factor que influye en su preparación académica, y 6 estudiantes, que representan el $3 \%$, no lo considera así. 


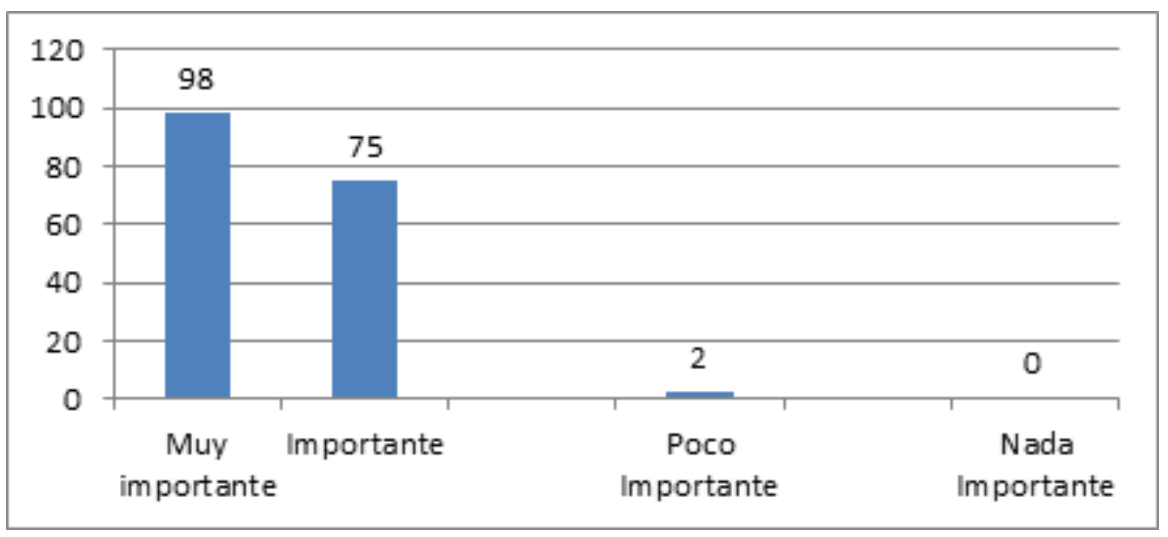

FIGURA 6

Evaluación global de los beneficios del software sobre las aptitudes académicas. Esquema elaborado para la investigación

En la Figura 6 se presenta la evaluación global de la muestra con respecto a los beneficios brindados por la herramienta desarrollada y su influencia en el desarrollo de las aptitudes académicas, se tiene que el $56 \%$ los califica como muy importante, el $43 \%$ los considera importante y apenas dos estudiantes, que representan el $1 \%$, creen que la herramienta es de poca importancia en este ámbito.

Con el fin de contrastar la evaluación del alumnado con el grado de aptitud académica real alcanzada, se aplicó una comparación de los puntajes obtenidos en las pruebas de pre y post estudio, cuyos resultados se presentan a continuación.

TABLA 1

Resultados comparativos por puntajes antes y después del uso del software desarrollado

\begin{tabular}{cccccccc}
\hline PUNTAJE & $\mathbf{0}$ & $\mathbf{1}$ & $\mathbf{2}$ & $\mathbf{3}$ & $\mathbf{4}$ & $\mathbf{5}$ & $\begin{array}{c}\text { TOTAL } \\
\text { DESPUÉS }\end{array}$ \\
\hline 0 & $\mathbf{0}$ & 0 & 0 & 0 & 0 & 0 & $\mathbf{0}$ \\
\hline 1 & 6 & $\mathbf{2}$ & 2 & 0 & 0 & 0 & $\mathbf{1 0}$ \\
\hline 2 & 10 & 12 & $\mathbf{3}$ & 0 & 0 & 0 & $\mathbf{2 5}$ \\
\hline 3 & 9 & 25 & 26 & $\mathbf{3}$ & 0 & 0 & $\mathbf{6 3}$ \\
\hline 4 & 3 & 15 & 16 & 13 & $\mathbf{9}$ & 0 & $\mathbf{5 6}$ \\
\hline 5 & 1 & 0 & 4 & 8 & 7 & $\mathbf{1}$ & $\mathbf{2 1}$ \\
\hline $\begin{array}{c}\text { TOTALES } \\
\text { ANTES }\end{array}$ & $\mathbf{2 9}$ & $\mathbf{5 4}$ & $\mathbf{5 1}$ & $\mathbf{2 4}$ & $\mathbf{1 6}$ & $\mathbf{1}$ & $\mathbf{1 7 5}$ \\
\hline
\end{tabular}

Para evaluar los resultados alcanzados, se generó la matriz que se presenta en la Tabla 1, en donde las columnas representan los puntajes obtenidos en la etapa pre estudio, y las filas representan los puntajes obtenidos en la etapa de post estudio, con la cantidad de estudiantes desplazados por notas luego de la aplicación.

En la tercera columna de la Tabla 1 se aprecia, por ejemplo, que 51 estudiantes obtuvieron una calificación de dos puntos en la prueba inicial, y luego de emplear el software lograron obtener una calificación de cinco 
en cuatro de los casos, 16 estudiantes obtuvieron una nota de cuatro, 26 estudiantes obtuvieron una nota de tres, tres estudiantes mantuvieron la nota y, como caso especial, dos estudiantes de toda la muestra estudiada bajaron su calificación.

Para 18 estudiantes, que se resaltan en la diagonal de la matriz expuesta, la herramienta no influyó en los resultados, pues mantienen el mismo puntaje. Se aprecia que 29 estudiantes que obtuvieron una calificación de cero lograron mejorar su nota, de los cuales, un sujeto obtuvo la máxima valoración. De toda la muestra, 21 estudiantes obtuvieron el máximo puntaje luego de la utilización de la herramienta, y se observa que no existen estudiantes con calificación de cero en la etapa de post estudio.

TABLA 2

Crecimiento de las calificaciones obtenidas en las etapas de pre y post estudio

\begin{tabular}{lcccccc}
\hline PUNTAJE & $\mathbf{0}$ & $\mathbf{1}$ & $\mathbf{2}$ & $\mathbf{3}$ & $\mathbf{4}$ & $\mathbf{5}$ \\
\hline $\begin{array}{l}\text { Pre-estudio } \\
\text { Post-estudio }\end{array}$ & $17 \%$ & $31 \%$ & $29 \%$ & $14 \%$ & $9 \%$ & $1 \%$ \\
$\begin{array}{l}\text { Crecimiento } \\
\text { porcentual }\end{array}$ & $-100 \%$ & $-81 \%$ & $-52 \%$ & $257 \%$ & $356 \%$ & $1200 \%$ \\
\hline
\end{tabular}

En la Tabla 2 se presenta el porcentaje de crecimiento de las calificaciones obtenidas en las etapas de pre y post estudio. Se aprecia que el porcentaje de crecimiento es mayor en la medida en que la calificación se acerca a la nota máxima: en la calificación de cero el crecimiento es negativo en un $100 \%$, mientras que, en la puntuación máxima, el crecimiento es de $1200 \%$.

Los resultados de la investigación determinan que existió un incremento promedio $409.2 \%$ en la puntuación. Las personas que obtuvieron bajas calificaciones (de cero a dos puntos) en el pre estudio incrementaron su calificación cuando se aplicó la herramienta software en un promedio de $77.67 \%$. Se destaca que todas las personas que obtuvieron una nota de cero, incrementaron su puntuación: 13 de ellas obtuvieron notas mayores o iguales a tres, y una alcanzó la nota de cinco.

Del grupo investigado, aquellos casos que en la etapa de pre estudio obtuvieron calificaciones mayores o iguales a tres, muestran un incremento promedio de $604.33 \%$, que es notablemente superior al grupo previamente analizado, y quince estudiantes desplazaron su calificación a la máxima puntuación.

Se observa que la herramienta software desarrollada no influye en la calificación alcanzada por 18 estudiantes que son el $9.72 \%$ de la muestra, de los cuales nueve estudiantes mantuvieron la nota de cuatro puntos. Como casos notables derivados de este análisis, se resalta que, de toda la muestra estudiada, dos estudiantes que obtuvieron una valoración de dos puntos en la etapa de pre estudio bajan su puntuación en la etapa de post estudio.

Tras el análisis de toda la muestra de investigación, se puede afirmar que el índice de impacto ejercido por la herramienta desarrollada es mayor mientras menor sea la calificación inicial obtenida: así, quienes obtuvieron notas menores que dos, incrementaron, en su mayoría, dos puntos; mientras que en los casos en que obtuvieron calificaciones iniciales de cuatro y cinco, en su mayoría mantuvieron la misma calificación.

Se resalta que en la etapa de pre estudio el mayor número de estudiantes (54) obtuvo una calificación de un punto, mientras que, en la etapa de post estudio, la mayor concentración, que son 63 estudiantes, se encuentra en la calificación de tres.

El grupo de estudiantes que formó parte de la investigación ratificó que el uso de la herramienta software de aptitud académica influyó en sus conocimientos (97\%), y atención (el $87 \%$ ) por las temáticas abordadas. El $84 \%$ de estudiantes afirma que la herramienta desarrollada no presenta problemas para su utilización por parte del público usuario. 
Para obtener el índice de crecimiento, se emplea el estadístico t para dos muestras emparejadas, es decir, cuando un sujeto es medido en dos condiciones diferentes, en este caso antes y después de la evaluación de la aptitud académica con la aplicación.

TABLA 3

Prueba t para media de dos muestras emparejadas

\begin{tabular}{lrr}
\hline & \multicolumn{1}{c}{ ANTES } & \multicolumn{1}{c}{ DESPUÉS } \\
\hline Media & 1,697142857 & 3,302857143 \\
Varianza & 1,442233169 & 1,08591133 \\
Observaciones & 175 & 175 \\
Coeficiente de correlación de Pearson & 0,606427565 & \\
Diferencia hipotética de las medias & 0 & \\
Grados de libertad & 174 \\
Estadístico t & $-21,13292164$ \\
$P(T<=t)$ una cola & $3,18889 \mathrm{E}-50$ \\
Valor crítico de t (una cola) & 1,411102093 \\
$P(T<=t)$ dos colas & $6,37778 \mathrm{E}-50$ & \\
Valor crítico de $t$ (dos colas) & 1,760970633 & \\
\hline
\end{tabular}

De la Tabla 3 se obtienen las puntuaciones de la media de pre estudio (\#a) que es 1,70 y el valor de la media de post estudio (\#d) de 3,30, con las que se obtiene 94,12\% como índice de crecimiento, esto indica que el software contribuye significativamente en el desarrollo de las aptitudes académicas.

\section{Conclusiones}

Se puede concluir que el empleo de un software educativo basado en estrategias didácticas favorece el proceso de aprendizaje y desarrollo de aptitudes académicas, ya que el $87 \%$ de personas encuestadas consideran que la integración de diversos tópicos en el software ayuda a mejorar la atención durante su empleo.

Al realizar una evaluación global de los beneficios de la aplicación informática sobre las aptitudes académicas, el $99 \%$ de la muestra considera que tiene un grado de importancia para este ámbito. Solo dos estudiantes consideran lo contrario, lo que se relaciona con el número de casos en los que no se presentaron mejoras en las calificaciones obtenidas, lo que amerita un análisis profundo desde ámbitos diferentes al tecnológico, tales como el entorno de estudio, motivación, aspectos psicológicos y los diferentes estilos de aprendizaje.

En la etapa de pre estudio, la mayor cantidad de estudiantes, equivalente al $30.86 \%$, obtuvo una calificación de un punto sobre cinco posibles, mientras que, en la etapa de post estudio, las mayores concentraciones se sitúan entre tres y cuatro puntos sobre cinco, con el $36 \%$ y $32 \%$ respectivamente, lo que refleja que la herramienta informática influyó positivamente en el proceso de evaluación, lo que redunda en el mejoramiento del aprendizaje.

La herramienta software desarrollada influyó en las calificaciones de la muestra; 155 estudiantes mejoraron su calificación, 18 estudiantes mantuvieron su calificación y en dos estudiantes la calificación disminuyó. Sin 
embargo, se evidencia que existe una relación inversa entre la calificación obtenida en la etapa de pre estudio y el beneficio ejercido por la herramienta software.

Al finalizar la investigación y obtenerse un crecimiento promedio de $94.12 \%$ en las calificaciones de la muestra, se determina que el empleo de herramientas software basadas en estrategias didácticas en el proceso de enseñanza aprendizaje pueden contribuir al fortalecimiento de este ámbito.

\section{REFERENCIAS}

Arancibia, V., Herrera, P., y Strasser, K. (2008). Manual de psicología educacional. Santiago de Chile: Universidad Católica de Chile.

Blanco, R., García, P., Gómez, C., Moreira, T., y Romero, E. (2016). Guía de la prueba de aptitud académica. Proceso de admisión 2015 - 2016. Tecnológico de Costa Rica. Recuperado de https://www.tec.ac.cr/sites/default/files/ media/doc/guia-_prueba_de_aptitud_academica_2015-2016.pdf

Bojórquez, J., López, L., Hernández, M. y Jiménez, E. (2014). Utilización del alfa de Cronbach para validar la confiabilidad de un instrumento de medición de satisfacción del estudiante en el uso del software Minitab. 11th Latin American and Caribbean Conference for Engineering and Technology, (pp. 1-9). Cancún. Recuperado de http://www.laccei.org/LACCEI2013-Cancun/RefereedPapers/RP065.pdf

Boza, A., y Toscano, M. (2012 de abril de 2012). Motivos, actitudes y estrategias de aprendizaje: Aprendizaje motivado en alumnos universitarios. Profesorado, 125 - 142. Obtenido de www.ugr.es/ recfpro/rev161ART8.pdf

Cataldi, Z. (2000). Metodología de diseño, desarrollo y evaluación de software educativo (Resumen). La Plata: Universidad de La Plata.

Chipia, J. (2008). Modelos de instrucción. Bogotá: Universidad de Los Andes.

ecuadorencifras.com. (2013). ecuadorencifras.com. Obtenido de http://www.ecuadorencifras.gob.ec/documentos/ web-inec/Estadisticas_Sociales/TIC/Resultados_principales_140515.Tic.pdf

Ecuadorencifras.gob.ec. (16 de mayo de 2014). 1,2 millones de ecuatorianos tienen un teléfono inteligente (Smartphone). Recuperado de http://www.ecuadorencifras.gob.ec/12-millones-de-ecuatorianos-tienen-untelefono-inteligente-smartphone/

El Universo. (25 de mayo de 2014). Alto puntaje, condicionante para lograr un cupo de ingreso a las universidades. Recuperdo de https://www.eluniverso.com/noticias/2014/05/25/nota/3006166/alto-puntaje-condicionantelograr-cupo-ingreso-u

El universo.com. (30 de noviembre de 2014). En dos años disminuyó el ingreso a la universidad en Ecuador. Recuperado de El universo: http://www.eluniverso.com/noticias/2014/11/30/nota/4284736/dosanos-disminuyo-ingreso-universidad

Fabila, A., y Izquierdo, J. (2013). La escala de Likert en la evaluación docente: Acercamiento a sus características y principios metodológicos. Perspectivas docentes, 50, 31-40. .Recuperado de file:///C:/Users/jrsc/Downloads/ FabMinIzq2012_Perspectivas_articulo.pdf

Fontalvo, H. (2008). Modelo de enseñanza - aprendizaje para un software educativo basado en la andragogía y el diseño instruccional de Robert Gagné. Núcleo de Informática en la educación especial (NIEE). Recuperado de www.ufrgs.br/niee/eventos/RIBIE/2008/pdf/modelo_ens_aprend_software.pdf

Gargallo, B., Pérez, C., Serra, B., Sánchez, F., y Ros, I. (25 de febrero de 2007). Actitudes ante el aprendizaje y rendimiento académico en los estudiantes universitarios. Revista Iberoamericana de Educación, 42, 1-25. Recuperado de http://webcache.googleusercontent.com/search?q=cache:u9F465Wf_6kJ:rieoei.org/ investigacion $/ 1537$ Gargallo.pdf $+\& c d=3 \& h l=e s-419 \& c t=c \operatorname{lnk} \& c l i e n t=$ firefox $-b$

Llaurado, O. (28 de marzo de 2011). La escala de Likert: Qué es y cómo utilizarla. Recuperado de http:// www.netquest.com/blog/es/la-escala-de-likert-que-es-y-como-utilizarla/

Llosent, M. (20 de marzo de 2015). FIKISMATICA. Blog Educativo. Recuperado de http:// fikismatica.blogspot.com/2015/03/practicas-para-el-examen-de-admicion-de.html 
Mantilla, F. (8 de septiembre de 2015). Técnicas de muestreo: Un enfoque a la investigación de mercados. Quito: Universidad de las Fuerzas Armadas ESPE. Obtenido de repositorio.espe.edu.ec/bitstream/21000/.../Técnicas \%20de\%20muestreo\%2C\%20.pdf

Morales, F. J. (22 de abril de 2015). Aptitudes y diferencias en estudiantes de tercero básico de Guatemala. Revista 22 UVG Universiada del Valle Guatemala. Recuperado de http://www.uvg.edu.gt/publicaciones/revista/ volumenes/numero-22/Revista_22_29-38.pdf

Peñalosa, E., y Castañeda, S. (2007). Meta-tutor: Construcción, descripción y aplicación de un ambiente de aprendizaje para internet. Revista Latinoamericana de Tecnología Educativa, 6(1). Recuperado de http:// campusvirtual.unex.es/cala/editio/

Secretaría de Educación Superior, Ciencia, Tecnología e Innovación. (Marzo de 2014). Puntajes referenciales mínimos y máximos de asignación. Recuperado de http://preuniversitarionewton.com/web/ona-dedescargas.html?....6:puntajes

Valencia, G. (Marzo de 2017). Razonamiento lógico numérico, lógico verbal y lógico abstracto en la educación. Journal of Education and Human Development, 89-95. Obtenido de http://jehdnet.com/journals/jehd/ Vol_6_No_1_March_2017/9.pdf

\section{Notas}

[5] DA/ED.- De acuerdo / En desacuerdo

\section{BY-NC-ND}

